\title{
A SET THEORETIC APPROACH TO IMAGE RECONSTRUCTION FROM PROJECTIONS
}

\author{
Bhaskar Bhumkar, Poorvi L. Vora, B. Chandna and K. Shankar \\ Department of Electrical Engineering \\ Indian Institute of Technology - Bombay \\ Powai, Mumbai 400076 (India) \\ poorvi@hpl.hp.com
}

\begin{abstract}
The reconstruction of an image from incomplete view data requires the use of several constraints not derived from ray sum (projection data) measurements. The constraints can be incorporated through the method of (sequential and parallel) projections onto the constraint sets. These methods for the use of information regarding the noise and the image are implemented and compared in this paper. It is shown that the use of noise statistics decreases the mean square error in the image and that the method of parallel projections results in smaller error than the method of sequential projections if a sufficient number of iterations is permitted.
\end{abstract}

\section{INTRODUCTION}

Algebraic methods were among the first used to solve the tomographic problem. These methods approach the tomographic problem as one where the solution is constrained to satisfy a set of linear equations. The algebraic reconstructive technique (ART) [1] seeks a solution by sequential projection of an initial estimate onto the sets of solutions of the linear equations, each of these sets is a hyperplane. The simultaneous iterative reconstructive technique (SIRT) [1] approaches the solution through parallel projection onto the hyperplanes and provides far better results in the presence of noise.

The method of sequential projections onto closed convex constraint sets for the general image recovery problem was proposed in [2] and implemented without using noise information on tomographic images in [3]. A set-theoretic framework for the use of prior information obtained from the noise statistics for the general signal recovery problem was provided in [4], and demonstrated to be very useful with certain types of

\footnotetext{
${ }^{0}$ Currently with Hewlett-Packard Laboratories, Palo Alto, CA, USA
}

signals. The use of (set-theoretic) parallel algorithms to solve the general constrained signal recovery problem was presented in [5], and these algorithms were shown to be more resilient to noise than (set-theoretic) sequential methods. The foundations of set-theoretic estimation are discussed in detail in [6], where the method of 'sequential projections is referred to as MOSP and that of parallel projections as MOPP.

This paper presents the use of parallel set-theoretic methods for the tomographic problem. It also suggests the use of noise information along the lines of [4] in the image reconstruction process. It is shown that the problems of noisy projection data can be partly overcome using MOPP (even without noise information) or MOSP with information about the noise variance.

\section{SET THEORETIC FORMULATION}

The tomographic problem may be formulated in settheoretic terms as follows. The linear model for image reconstruction may be written as,

$$
\mathbf{g}=\mathbf{H f}+\mathbf{n}
$$

where $\mathbf{g}$ is the projection data vector of size $(M \times 1)$, $\mathbf{f}$ is the original image vector of size $\left(N^{2} \times 1\right), \mathbf{H}$ is the weight matrix of size $\left(M \times N^{2}\right), \mathbf{n}$ is the signalindependent, gaussian, zero-mean noise vector of size $(M \times 1)$.

The required image, $\tilde{\mathbf{f}}$, is assumed square. $\mathbf{f}(i)$ represents the $i^{t h}$ pixel of any image $\mathrm{f} \in \Re^{N \times N}$. The residual [4], $\mathbf{r}=\mathbf{g}-\mathbf{H} \tilde{\mathbf{f}}$, is the 'noise estimate'. Constraints on the residual may be expressed as constraints on the required image. The required image may be chosen to belong to one or many of the following constraint sets in the $N^{2}$-dimensional vector space over the reals:

1. Set of all images with non-negative pixels

$$
C_{1}=\{\mathbf{f} \text { s.t.f }(i) \geq 0 \forall i\}
$$


2. Set of all images whose pixel values lie in a prescribed closed interval;

$$
C_{2}=\{\mathbf{f} s . t . \alpha \leq \mathbf{f}(i) \leq \beta \forall i\}
$$

3. Set of images with known points,

$$
C_{3}=\left\{\mathbf{f} s . t . \mathbf{f}(i)=\gamma_{i} \forall i \in I \subset\left\{1 \ldots . N^{2}\right\},\right\}
$$

4. Set of all images that provide a residual of reasonable mean [4]

$$
C_{m}=\left\{\mathbf{f}|| \sum_{i}\left(\mathbf{g}_{i}-[\mathbf{H} \mathbf{f}]_{i}\right) \mid \leq \delta_{m}\right\}
$$

where $\mathbf{g}_{i}$ and $[\mathbf{H f}]_{i}$ are the $i^{\text {th }}$ elements of vectors $\mathrm{g}$ and $\mathbf{H f}$ respectively. The bound $\delta_{m}$ represents the confidence limits on the sample mean. The size of the set is determined by $\delta_{m}$.

5. Set of all images that provide a residual of reasonable variance [4]

$$
C_{v}=\left\{\mathbf{f} \mid\|\mathbf{g}-\mathbf{H f}\|^{2} \leq \delta_{v}\right\}
$$

The bound $\delta_{v}$ represents the confidence limits on the sample variance. The size of the set is determined by $\delta_{v}$.

It is simple to check that each of the above sets is convex and closed. In MOSP, an initial estimate is projected onto each of the above closed convex sets in a cyclic fashion. In MOPP, all sets are activated at each iteration, i.e., projections are made on all sets during each iteration [5]. The estimate is a weighted-average of all the projections. Both methods converge to a point in the intersection of the constraint sets if the intersection is non-empty. If the intersection is empty, MOSP does not converge and oscillates among the constraint sets. MOPP, however, converges to a minimum weigthed-square solution, i.e. it converges to a point whose distances from the constraint sets are such that the weighted sum of the squares of these distances is minimum [6].

In the image reconstruction problem, nonintersecting constraint sets often arise because of noisy projection data which gives rise to a set of inconsistent linear equations. ART and SIRT are specific cases of MOSP and MOPP respectively, when the only constraint sets are the hyperplanes defined by the projection data. It is well-known that SIRT has far better noise characteristics than does ART. ART deals with noise by allowing variation in the hyperplane, but this treats each noise variable separately. Using the sets $C_{v}$ and $C_{m}$ in addition to the constraints used for ART will allow a method in which the noise can be treated as a random process.

Illustrative results that demonstrate the use of constraint sets other than non-negativity and the ray-sum linear equations are presented in the next section. The next section also presents an example of the better behaviour of MOPP in noisy conditions.

\section{SIMULATIONS AND RESULTS}

Simulations were mainly carried out on three images : (i) An elliptical image of size $10 \times 10$ with uniform grey level in the background, with 130 equations in 100 unknowns.

(ii) A sagittal section of the human brain of size $64 \times$ 64, 5520 equations in 4096 unknowns.

(iii) A $16 \times 16$ image of alternating white and black squares of value 0 and 255 .

Apart from these images another image of the human head was used to confirm the results.

Ray sums were calculated and simulated zero-mean, gaussian, independent, identically distributed noise variables added.

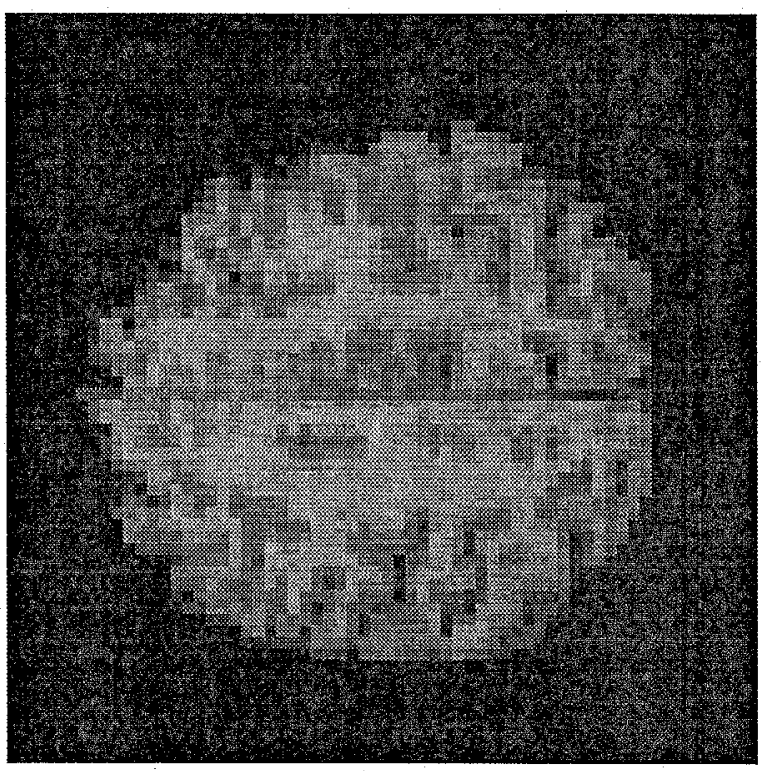

Figure 1: Original Image.

Fig. 1 shows the original $64 \times 64$ brain image. Fig. 2 shows its reconstruction using ART. The mean-square error in this case is 117.61 , corresponding to an RMS error of 10.84. The maximum value for the image is 255. Fig. 3 shows the reconstructed image with use of $C_{2}$, the set of images with pixels bounded above by 255 and below by 0 , in MOSP. The mean square error 
in this case is 80.97 , corresponding to an RMS error of almost 9 .

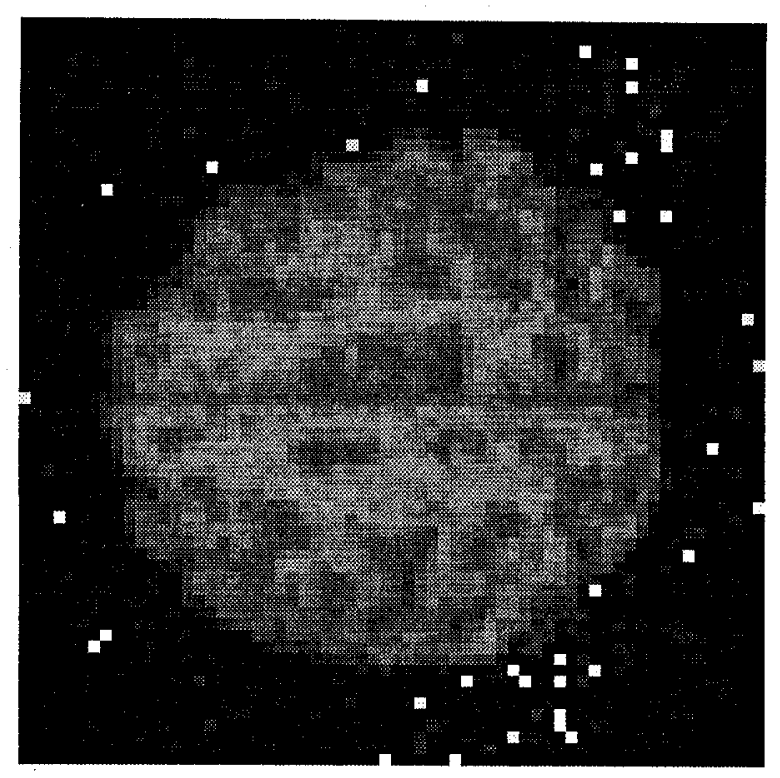

Figure 2: ART Reconstruction.

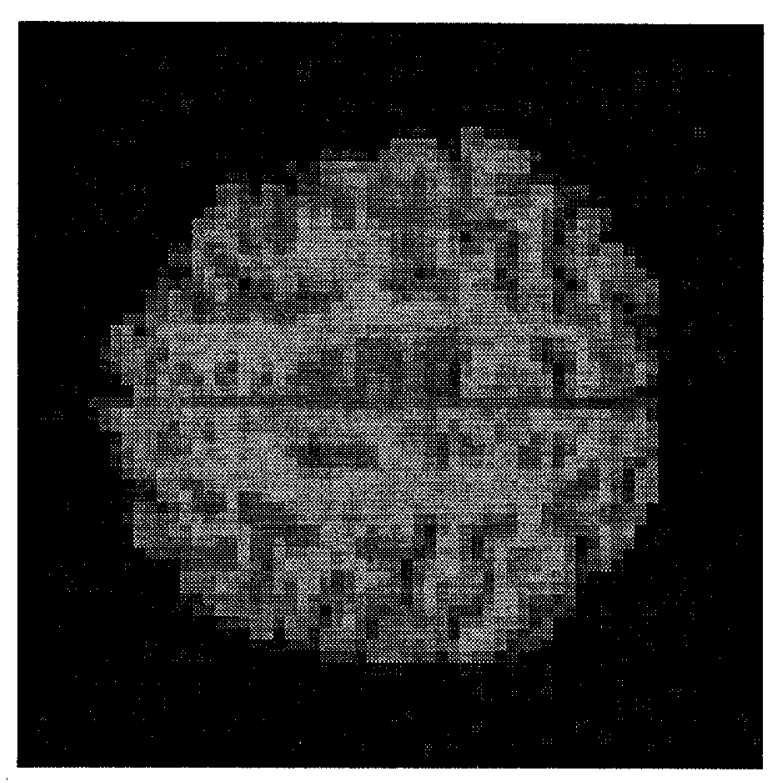

Figure 3: MOSP Reconstruction with $C_{v}$.

The set of images with bounded pixels is very effective since the mean square error has reduced remarkably. The above images are representative and the reduction in error is true for both noisy as well as non- noisy cases. The above error-reduction was also noted in ART with non-negativity, but the above formulation provides far more flexibility.

Gaussian noise of mean 0 and variance 1 is added to the projection data and the image reconstructed using $C_{v}$, the residual variance set, in addition to the linear equations and non-negativity. Table 1 lists results for projections including $C_{v}$ for the ellipse image. MSE1 is the mean square error for $\delta_{v}=2.2493$, and MSE2 is the mean square error for $\delta_{v}=1.7649$. Lagrange's multipliers for the projection operator are found by solving the constraint equation by the Newton-Raphson method [4]. Projecting onto $C_{v}$ is computationally highly intensive [4].

Table 1: MSE for Projections onto $C_{v}$, for ellipse.

\begin{tabular}{|c|c|r|r|}
\hline \#Iterations & $\begin{array}{c}\text { Newton } \\
\text { Iterations }\end{array}$ & MSE1 & MSE2 \\
\hline 50 & 2 & 0.25 & 0.18 \\
\hline 20 & 2 & 0.25 & 0.18 \\
\hline 10 & 2 & 0.32 & 0.24 \\
\hline 10 & 5 & 0.15 & 0.13 \\
\hline
\end{tabular}

The size of the set plays an important role during reconstruction. As the size of the set decreases, error also decreases. This is to be expected and explains the pattern of error observed in Table 1. Further, for the $10 \times 10$ image, the MSE using $C_{1}$ is 0.005084 , while using the set $C_{2}$, of images whose pixels are bounded above by 1 and below by 0 , the reconstruction is almost perfect. The first set is unbounded, the second is bounded.

The $16 \times 16$ checker-board image was reconstructed using 1) ART and non-negativity, 2) ART, non-

negativity and the noise variance constraint as MOSP, and 3 ) the noise variance constraint. The MOSP results of 2) were far better than the those of 1 ), and the results of 3 ) were comparable to those of 1). This indicates the usefullness of the variance constraint.

MOSP works quite well in non-noisy data situations, but its performance deteriorates in noisy situations when the variance constraint is not used. MOPP performs well in the presence of noise without the variance constraint, but it requires a large number of iterations to assure convergence. This is evident from the following graph (Fig. 4) for the ellipse.

After 50 iterations the MSE in MOPP is less than that in MOSP. The main disadvantage of MOPP, however, is its poor convergence rate. For the $64 \times 64$ brain image, the mean square difference between consecutive iterates for 30 iterations is 0.00954 in MOSP 
and 0.063154 in MOPP. The final solution for MOSP depends upon the sequence with which the projections are made onto the sets. This is not the case with MOPP where the projections are made on all the sets during each iteration and the weighted average of all projections is considered as the next estimate.

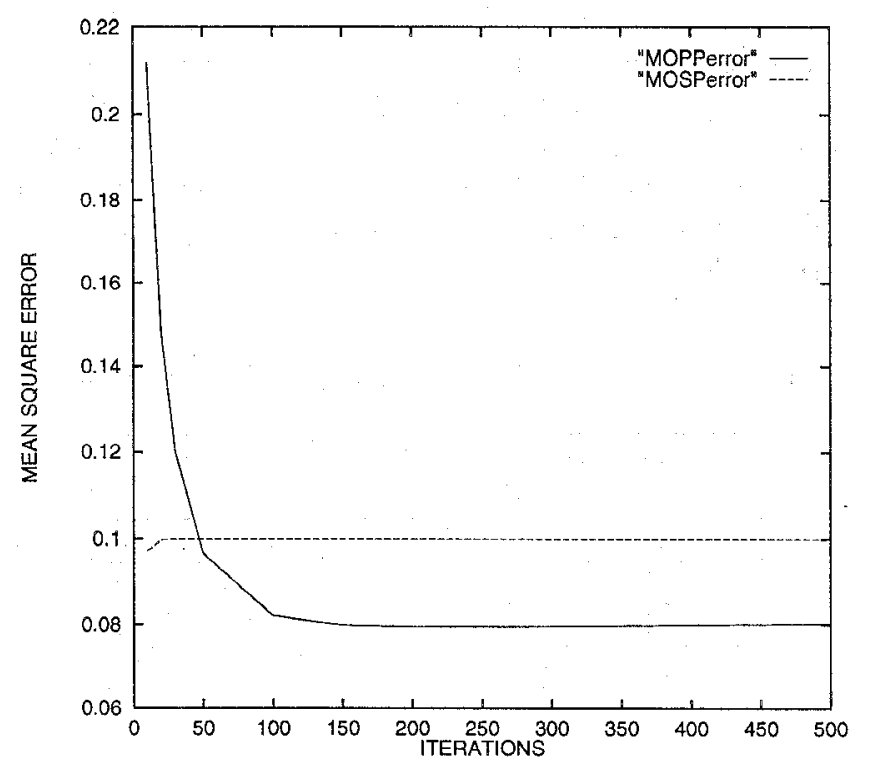

Figure 4: MSE V/s No of iterations in MOSP and MOPP for projection data with noise.

\section{CONCLUSIONS}

The use of MOSP and MOPP for tomographic reconstruction has been demonstrated. The methods provide means of using information of the noise statistics to reconstruct images from noisy projection data. While more computationally intense than the non-iterative methods, these methods provide a means of incorporating prior information into the reconstruction procedure that the non-iterative methods do not.

\section{REFERENCES}

[1] Herman G. T., Image Reconstruction from Projections: The Fundamentals of Computerised Tomography, New York Academic, 1980.

[2] Youla D.C. and Webb H., "Image restoration by method of convex projections : part-1 Theory", IEEE Trans. Medical Imaging, Vol. MI-1, No. 2, pp. 81-94, Oct. 1982.

[3] Sezan M.I. and Stark H., "Image restoration by method of convex projections : part-2 Applications and numerical results", IEEE Trans. Medical Imaging, Vol. MI-1, No. 2, pp. 95-101, Oct. 1982.

[4] Trussell H. and Civanlar M.R., "The feasible solution in signal restoration", IEEE Trans. Acoustics, Speech and Signal Processing, Vol. ASSP - 32, No. 2, pp. 201-212, Apr. 1984.

[5] Combettes P.L. and Puh H., "Parallel projection methods for set theoretic signal reconstruction and restoration", Proc. IEEE Int. Conf. on Acoustics, Speech and Signal Processing, Minneapolis, MN, Vol. 5, Apr. 2730, 1993, pp. 297-300.

[6] Combettes P.L., "The foundations of set theoretic estimation", Proc.IEEE, Vol. 81, No. 2, pp. 182-208, Feb. 1993. 